\title{
Differential form representation of stochastic electromagnetic fields
}

\author{
Michael Haider and Johannes A. Russer \\ Institute for Nanoelectronics, Technical University of Munich, Arcisstraße 21, 80333 München, Germany \\ Correspondence to: Michael Haider (michael.haider@tum.de)
}

Received: 23 December 2016 - Revised: 25 February 2017 - Accepted: 27 February 2017 - Published: 21 September 2017

\begin{abstract}
In this work, we revisit the theory of stochastic electromagnetic fields using exterior differential forms. We present a short overview as well as a brief introduction to the application of differential forms in electromagnetic theory. Within the framework of exterior calculus we derive equations for the second order moments, describing stochastic electromagnetic fields. Since the resulting objects are continuous quantities in space, a discretization scheme based on the Method of Moments (MoM) is introduced for numerical treatment. The MoM is applied in such a way, that the notation of exterior calculus is maintained while we still arrive at the same set of algebraic equations as obtained for the case of formulating the theory using the traditional notation of vector calculus. We conclude with an analytic calculation of the radiated electric field of two Hertzian dipole, excited by uncorrelated random currents.
\end{abstract}

\section{Introduction}

The most widely used concept for the formulation of Maxwell's equations is the vector field approach. Even though vector calculus became a quasi-standard for engineering applications, other formulations like tensor calculus, quaternions and differential forms could provide deeper insight into the underlying physics (Warnick and Russer, 2014). The theoretic treatment for stochastic electromagnetic fields, originating from noise sources with Gaussian probability distribution, has been given by Russer and Russer (2015), using the traditional notation of vector calculus.

Characterization and modeling of stationary stochastic electromagnetic fields using field correlations has been expanded to the transmission line matrix method in Russer et al. (2016), to noisy cyclostationary fields (Russer et al., $2015 b, c$ ), and it has been used for source localization (Gorbunova et al., 2013; Baev et al., 2013; Kuznetsov et al.,
2016) and imaging of radiated electromagnetic interference (EMI) sources (Russer and Russer, 2011b). Free space electromagnetic field propagation considering field-field correlations has been addressed in (Gradoni et al., 2015). Near field measurements for radiated EMI is discussed in (Russer et al., 2015d), while analysis of measurement data by principal component analysis, critical for large data sets acquired in characterizing fields by correlation information, is discussed in (Arnaut and Obiekezie, 2014; Haider and Russer, 2017).

As in the case of deterministic fields, it can be expected that differential forms may lead to a better understanding of stochastic electromagnetic fields. Within this work, we take advantage of differential form representation for the modeling of noisy processes. Noise is an inevitable perturbation in wireless communication scenarios. While noise is a stochastic process also interfering signals originating from deterministic processes may have to be treated as quasi-stochastic signals due to lack of knowledge or the prohibitive complexity to model the deterministic process. Accurate noise modeling is crucial with respect to electromagnetic compatibility (EMC), electromagnetic interference (EMI), and signal integrity (SI) considerations for the design of electronic components and systems. A careful modeling of noisy processes and stochastic electromagnetic fields shows also potential to improve wireless- and on-chip communication (Nossek et al., 2013).

\section{Differential forms}

The calculus of exterior differential forms was introduced by Élie Cartan (1945), based on previous work from Hermann Grassmann, who himself introduced the exterior algebra in (Grassmann and Kannenberg, 1995). The exterior algebra is based upon the exterior product,

$a \wedge b=-b \wedge a$, 
which is defined in more detail in Russer (2006, p. $631 \mathrm{ff})$ as well as in Warnick and Russer $(2014,2006)$. We begin with introducing the fundamental elements of the mathematical framework of exterior calculus. Let $\boldsymbol{A}: U \subseteq \mathbb{R}^{3} \rightarrow \mathbb{R}^{3}$ be a vector field. We consider the line integral of $\boldsymbol{A}$ along a piecewise smooth curve $C \subset U$, given by

$$
\int_{C} \boldsymbol{A}(\boldsymbol{r}) \cdot \mathrm{d} \boldsymbol{r} .
$$

The dot-product in Eq. (2) assigns each element of $\boldsymbol{A}$ a differential for the respective direction in $\mathbb{R}^{3}$. By including these differentials into the vector field itself, a new quantity is introduced. With the choice of a certain basis in $\mathbb{R}^{3}$, this quantity can be expressed by its coefficients,

$\mathcal{A}=A_{x} \mathrm{~d} x+A_{y} \mathrm{~d} y+A_{z} \mathrm{~d} z$.

Using this expression, the integral from Eq. (2) can be rewritten as

$\int_{C} \mathcal{A}$.

The quantity $\mathcal{A}$ is called differential form. It is of degree one, i.e. it can be integrated over a curve $C$, which is a function possessing one degree of freedom. Differential forms of degree two and three are introduced in the same way, where a two-form is a quantity which can be integrated over area, while a three-form is integrated over volume. For the definition of two- and three-forms we make use of the exterior product, shown in Eq. (1). The exterior product accounts for the orientation of a surface in a natural way, when we integrate a two-form over an area. Two-forms, often referred to as bivectors (Lindell, 2004), are themselves dual to a corresponding one-form. This duality is reflected by means of the Hodge star operator $\star$. One can say that the star operator takes a differential form and converts it to a new differential form consisting of the differentials missing in the old form (Warnick and Russer, 2014). The action of the Hodge dual for one-forms and two-forms is given by

$$
\begin{array}{ll}
\star \mathrm{d} x=\mathrm{d} y \wedge \mathrm{d} z & \star \mathrm{d} y \wedge \mathrm{d} z=\mathrm{d} x, \\
\star \mathrm{d} y=\mathrm{d} z \wedge \mathrm{d} x & \star \mathrm{d} z \wedge \mathrm{d} x=\mathrm{d} y, \\
\star \mathrm{d} z=\mathrm{d} x \wedge \mathrm{d} y & \star \mathrm{d} x \wedge \mathrm{d} y=\mathrm{d} z,
\end{array}
$$

and for the three- and zero-form by

$\star \mathrm{d} x \wedge \mathrm{d} y \wedge \mathrm{d} z=1$.

A one-form is naturally dual to a vector in $\mathbb{R}^{3}$. As long as Cartesian coordinates are used, the components of the dual vector are just given by the coefficients of the respective differential form. This duality relation changes, for different coordinate systems. We see that Eq. (4) keeps its form, irrespective of the considered coordinate system. Only the components of the dual vectors must be evaluated for each coordinate system separately, if a representation by components is needed. When considering electromagnetic fields, the notation becomes completely independent of the choice of a specific coordinate system, an advantage of differential forms over vectors. Furthermore, Warnick and Russer (2014) presented a very intuitive way for visualizing one-, two- and three-forms.

Maxwell's equations are often given in terms of differential equations, incorporating "curl" and "div" operators in traditional vector calculus notation. This representation can be generalized to differential forms, by introducing the exterior differential operator " $\mathrm{d}$ ". The exterior differential operator acting on an $n$-form $\mathcal{A}$ yields a new $(n+1)$-form, representing the spatial variations of $\mathcal{A}$. In a three-dimensional Cartesian coordinate system, the exterior derivative operator has the representation,

$\mathrm{d}=\left(\frac{\partial}{\partial x} \mathrm{~d} x+\frac{\partial}{\partial y} \mathrm{~d} y+\frac{\partial}{\partial z} \mathrm{~d} z\right) \wedge$,

where the differentials of the operator are connected with those of the differential form by the exterior product, while the partial derivatives act on the respective coefficients.

With the framework presented so far, we can postulate Maxwell's equations in a very general and coordinate independent way, starting with Gauss' law, which relates an electric displacement field $\mathcal{D}$ to a charge density $\mathcal{Q}$. The charge density $\mathcal{Q}$ is represented by a form of degree three, since it is a quantity which can be integrated over volume. We call $\mathcal{Q}$ closed, since $\mathrm{d} \mathcal{Q}=0$. This fact can be easily verified by using Eqs. (1) and (9). Poincaré's lemma (Lang, 1999, p. $137 \mathrm{ff}$ ), then ensures the existence of a two-form $\mathcal{D}$, such that $\mathrm{d} \mathcal{D}=\mathcal{Q}$. By this, Gauss' law is a direct consequence of how we describe fields by differential forms. Altogether, time harmonic Maxwell's equations expressed using differential forms are given by

$$
\begin{aligned}
\mathrm{d} \mathcal{E} & =-j \omega \mathcal{B}, \\
\mathrm{d} \mathcal{H} & =\mathcal{J}+j \omega \mathcal{D}, \\
\mathrm{d} \mathcal{D} & =\mathcal{Q}, \\
\mathrm{d} \mathcal{B} & =0 .
\end{aligned}
$$

Compared to vector calculus notation, we do not have the "div" and "curl" operators within Maxwell's equations. Hence, we unified their form by introducing exterior calculus to electromagnetics.

\section{Stochastic electromagnetic fields}

As pointed out by Russer and Russer (2015), noise has to be modeled as a stochastic electromagnetic field. Under the assumption that the considered fields can be assumed as Gaussian stationary random processes, it suffices to focus on the first and second order moments (Russer and Russer, 2015). 
The first order moment, i.e. the mean, can be set to zero without loss of generality. The second order moments are described by auto- and cross-correlation spectra. Consider a random current density $\mathcal{J}$ representing the source of a stochastic electromagnetic field. We obtain the electric field $\mathcal{E}$, excited from $\mathcal{J}$ by the integral

$\mathcal{E}=\int_{V^{\prime}} \mathcal{G} \wedge \mathcal{J}^{\prime}$

with a suitable Green's double one-form $\mathcal{G}$ (Russer, 2006, p. 129). The Green's double one-form relates the source current $\mathcal{J}^{\prime}$ in primed coordinates, i.e. the domain where $\mathcal{J}^{\prime}$ is non-vanishing, to the electric field $\mathcal{E}$ in the observation domain (Warnick and Arnold, 1996). The integral is extended over the complete volume of the source domain in primed coordinates. In order to represent the second order moments of the electric field $\mathcal{E}$ and source current $\mathcal{J}$, we define autoand cross-correlation functions of random signals $\left\{s_{i}\right\}_{i=1}^{N}$ in time-domain by

$c_{i j}(\tau)=\int_{-\infty}^{\infty} s_{i}(t) s_{j}(t-\tau) \mathrm{d} t$.

We call $c_{i i}(\tau)$ auto-correlation function for $i=j$ and crosscorrelation function for $i \neq j$. It should be pointed out here, that a stationary random process is not square integrable over time in general. Therefore, one needs to introduce timewindowed quantities in order to properly define frequency domain variables by Fourier transform. We treat each spatial component of $\mathcal{E}_{T}$ and $\mathcal{J}_{T}$ as the Fourier transform of a time-windowed stationary random processes with Gaussian statistics. For a compact notation, subscripts ${ }_{1}$ and ${ }_{2}$ denote the dependency on different spatial coordinates, $\boldsymbol{r}_{1}$ and $\boldsymbol{r}_{2}$, while a subscript ${ }_{T}$ indicates time-windowing. The correlation double one-form for the electric field, and the double two-form for the current density are given by

$$
\begin{aligned}
& \boldsymbol{\Gamma}_{E}\left(\boldsymbol{r}_{1}, \boldsymbol{r}_{2}, \omega\right)=\lim _{T \rightarrow \infty} \frac{1}{2 T}\left\langle\mathcal{E}_{1 T} \otimes \mathcal{E}_{2 T}^{*}\right\rangle, \\
& \boldsymbol{\Gamma}_{J}\left(\boldsymbol{r}_{1}, \boldsymbol{r}_{2}, \omega\right)=\lim _{T \rightarrow \infty} \frac{1}{2 T}\left\langle\mathcal{J}_{1 T} \otimes \mathcal{J}_{2 T}^{*}\right\rangle,
\end{aligned}
$$

where the brackets $\langle\cdot\rangle$ denote the forming of an ensemble average, the star superscript ${ }^{*}$ means complex conjugate, and $\otimes$ is an implied tensor product. By inserting Eq. (14) into Eq. (16), we obtain the correlation double one-form of the electric field from the correlation double two-form of the source currents,

$$
\boldsymbol{\Gamma}_{E}\left(\boldsymbol{r}_{1}, \boldsymbol{r}_{2}, \omega\right)=\iint_{V_{1}^{\prime} V_{2}^{\prime}} \mathcal{G}_{1} \wedge \boldsymbol{\Gamma}_{J}\left(\boldsymbol{r}_{1}^{\prime}, \boldsymbol{r}_{2}^{\prime}, \omega\right) \wedge \mathcal{G}_{2}^{*} .
$$

Similar results have been obtained in (Russer and Russer, 2015, 2011d, a, c; Russer et al., 2014), however, with traditional vector calculus notation.

\section{Method of moments}

The correlation double one-forms and two-forms for the electric field and the source currents, respectively are continuous in space. In order to enable a numerical treatment of problems related to stochastic electromagnetic fields, a discretization scheme has to be introduced. We use the method of moments (MoM) to transform field problems to network problems following (Russer and Russer, 2011a; Felsen et al., 2009; Harrington, 1968). The MoM is based on expanding an unknown function $f$ into a series of known basis functions $\left\{u_{n}\right\}_{n=1}^{N}$ with unknown coefficients. The dimension of the problem needs to be truncated after a finite $N \in \mathbb{N}$ in order to facilitate numerical evaluation on a computer. The goal is to establish a linear equation, relating the unknown series coefficients to known source coefficients. So let $f$ be an unknown function which is mapped to a known function $g$ by a linear operator $\mathcal{L}$,

$\mathcal{L}(f)=g$.

By developing $f$ into a series of basis functions $\left\{u_{n}\right\}_{n=1}^{N}$, we obtain

$\sum_{n=1}^{N} a_{n} \mathcal{L}\left(u_{n}\right)=g$.

Note that so far, we did not make any approximations, if $\left\{u_{n}\right\}_{n=1}^{N}$ forms a complete basis. Also the known function $g$ can be expanded by a sum over a set of so called weighting functions $\left\{w_{m}\right\}_{m=1}^{N}$,

$g=\sum_{m=1}^{N} b_{m} w_{m}$

where the coefficients $b_{m}$ are obtained by the inner product

$b_{m}=\left\langle w_{m}, g\right\rangle$.

After the second series expansion, the final problem reads as

$\sum_{n=1}^{N} a_{n}\left\langle w_{m}, \mathcal{L}\left(u_{n}\right)\right\rangle=\left\langle w_{m}, g\right\rangle$,

for each index $m \leq N$. By summarizing the coefficients $\left\{a_{n}\right\}_{n=1}^{N}$ and $\left\{\left\langle w_{m}, g\right\rangle\right\}_{m=1}^{N}$ into vectors, we arrive at our desired system of linear equations, relating unknown coefficients to source coefficients,

$\left[\begin{array}{ccc}\left\langle w_{1}, \mathcal{L}\left(u_{1}\right)\right\rangle & \cdots & \left\langle w_{1}, \mathcal{L}\left(u_{N}\right)\right\rangle \\ \left\langle w_{2}, \mathcal{L}\left(u_{1}\right)\right\rangle & \cdots & \left\langle w_{2}, \mathcal{L}\left(u_{N}\right)\right\rangle \\ \vdots & \ddots & \vdots \\ \left\langle w_{N}, \mathcal{L}\left(u_{1}\right)\right\rangle & \cdots & \left\langle w_{N}, \mathcal{L}\left(u_{N}\right)\right\rangle\end{array}\right]\left[\begin{array}{c}a_{1} \\ a_{2} \\ \vdots \\ a_{N}\end{array}\right]=\left[\begin{array}{c}\left\langle w_{1}, g\right\rangle \\ \left\langle w_{2}, g\right\rangle \\ \vdots \\ \left\langle w_{N}, g\right\rangle\end{array}\right]$. 
After addressing some fundamentals of the differential form representation of the electromagnetic field we proceed to apply the method of moments as outlined above. First of all, we need to define a proper inner product for imposing Hilbert space structure in our solution domain. Let our solution domain be $U \subseteq \mathbb{R}^{3}$ and let $\omega$ and $v$ be differential forms of the same degree $D \in\{1,2\}$ on $U$. We define an inner product for one-forms and two-forms on $U$ by

$$
\langle\omega, v\rangle=\int_{U} \omega^{*} \wedge \star v .
$$

This definition satisfies the requirements for an inner product (Lang, 1999), as we will verify in the following. Let $a, b \in \mathbb{C}$ be constants, and let $\omega, \mu, v$ be differential forms of degree $D \in\{1,2\}$. Then the following holds,

$$
\begin{aligned}
\langle\omega+\mu, v\rangle & =\int_{U}(\omega+\mu)^{*} \wedge \star v \\
& =\int_{U} \omega^{*} \wedge \star v+\int_{U} \mu^{*} \wedge \star v, \\
\langle\omega, a v\rangle & =\int_{U} a \omega^{*} \wedge \star v=a \int_{U} \omega^{*} \wedge \star v=a\langle\omega, v\rangle, \\
\langle\omega, v\rangle & =\int_{U} \omega^{*} \wedge \star v=\int_{U} v \wedge \star \omega^{*}=\langle\nu, \omega\rangle^{*} .
\end{aligned}
$$

The last requirement for a valid inner product is that it has to be a positive semi-definite functional. Since $\omega$ is either a one-form or a two-form, $\star \omega$ has exactly the opposite degree. For showing positive semi-definiteness, we choose $\omega$ to be a one-form, without loss of generality. The Hodge dual $\star \omega$ is therefore given as a two-form, with the same coefficients as the one-form $\omega$. We proceed by expressing $\omega$ by a sum over its components. Hence, we have

$$
\begin{aligned}
\langle\omega, \omega\rangle & =\int_{U} \omega^{*} \wedge \star \omega \\
& =\int_{U}\left(\sum_{n} \omega_{n} \mathrm{~d} x_{n}\right) \wedge\left(\frac{1}{2} \sum_{i, j} \omega_{i j} \mathrm{~d} x_{i} \wedge \mathrm{d} x_{j}\right),
\end{aligned}
$$

where $\omega_{i j}$ is an anti-symmetric tensor, i.e. $\omega_{i j}=-\omega_{j i}$. The coefficients $\omega_{i j}$ are related to the coefficients $\omega_{n}$, by

$\omega_{1}=\omega_{23}=-\omega_{32}$,

$\omega_{2}=\omega_{31}=-\omega_{13}$,

$\omega_{3}=\omega_{12}=-\omega_{21}$.

as can be seen from Eqs. (5)-(7). For $n=i, n=j$, or $i=j$ we do not get any contribution, since $\mathrm{d} x_{i} \wedge \mathrm{d} x_{i}=0$. There are only contribution, if $n \neq i \neq j$ holds. Thus, we get

$\langle\omega, \omega\rangle=\int_{U}\left(\omega_{1}^{*} \omega_{23} \mathrm{~d} x_{1} \wedge \mathrm{d} x_{2} \wedge \mathrm{d} x_{3}\right.$

$$
\begin{aligned}
& \left.+\omega_{2}^{*} \omega_{13} \mathrm{~d} x_{2} \wedge \mathrm{d} x_{1} \wedge \mathrm{d} x_{3}+\omega_{3}^{*} \omega_{12} \mathrm{~d} x_{3} \wedge \mathrm{d} x_{1} \wedge \mathrm{d} x_{2}\right) \\
& =\int_{U}\left(\left|\omega_{1}\right|^{2}+\left|\omega_{2}\right|^{2}+\left|\omega_{3}\right|^{2}\right) \mathrm{d} x_{1} \wedge \mathrm{d} x_{2} \wedge \mathrm{d} x_{3}
\end{aligned}
$$

where we used the Hodge duality relations given in Eqs. (5)(7). Since each contribution is non-negative, we can conclude that

$0 \leq\langle\omega, \omega\rangle$

where $\langle\omega, \omega\rangle=0$ only holds, if $\omega=0$. We have defined the zero-element to be a form with all coefficients equal to 0 .

In the next step, we expand the electric field $\mathcal{E}$ and the current density $\mathcal{J}$ into basis elements. Since $\mathcal{E}$ and $\mathcal{J}$ are differential forms, the basis elements also need to be oneforms and two-forms, respectively. The series expansions are given as

$$
\begin{aligned}
& \mathcal{E}=\sum_{n=1}^{N} V_{n}(\omega) \mathcal{U}_{n}, \\
& \mathcal{J}=\sum_{n=1}^{N} I_{n}(\omega) \mathcal{V}_{n},
\end{aligned}
$$

where the basis one-forms $\mathcal{U}_{n}$ for the electric field and the basis two-forms $\mathcal{V}_{n}$ for the current density are of the same family, i.e. $\mathcal{U}_{n}=\star \mathcal{V}_{n}$. This choice can be interpreted as the differential forms version of Galerkin's method (Gibson, 2008). The differential form bases are chosen in such a way, that the orthogonality relation

$\left\langle\mathcal{U}_{m}, \mathcal{U}_{n}\right\rangle=\int_{V} \mathcal{U}_{m}^{*} \wedge \star \mathcal{U}_{n}=\delta_{m n}$,

holds. In practice, this is quite uncommon since numerical solvers utilizing the method of moments often use triangular or sinusoidal basis functions with an extent over several mesh cells. Nevertheless, we use this restriction in order to make the following equations more readable. The coefficients $V_{n}$ and $I_{n}$ can be considered as generalized voltages and generalized currents (Russer and Russer, 2011c). Inserting the series expansions (35) and (36) into the electric field integral Eq. (14) and applying the inner product (25) yields

$$
V_{m}(\omega)=\sum_{n=1}^{N} I_{n}(\omega) \int_{V} \int_{V^{\prime}} \mathcal{V}_{m}^{*} \wedge \mathcal{G} \wedge \mathcal{V}_{n}^{\prime}
$$

We can identify the MoM matrix elements by

$$
Z_{m n}(\omega)=\iint_{V} \mathcal{V}_{V^{\prime}}^{*} \wedge \mathcal{G} \wedge \mathcal{V}_{n}^{\prime},
$$


and simplify the Eq. (38) to

$V_{m}(\omega)=\sum_{n=1}^{N} I_{n}(\omega) Z_{m n}(\omega)$.

The same result has been obtained in Russer and Russer (2011c), but with traditional vector calculus notation. Since differential forms are naturally dual to vectors, and a double one-form can be expressed by a dyadic function in a chosen coordinate system, it can be argued that both results are indeed equal.

We can now calculate correlation matrices for the generalized voltages $V_{n}(\omega)$ and the generalized currents $I_{n}(\omega)$,

$$
\begin{aligned}
C_{V, m n}(\omega) & =\lim _{T \rightarrow \infty} \frac{1}{2 T}\left\langle V_{m T}(\omega) V_{n T}^{*}(\omega)\right\rangle, \\
C_{I, m n}(\omega) & =\lim _{T \rightarrow \infty} \frac{1}{2 T}\left\langle I_{m T}(\omega) I_{n T}^{*}(\omega)\right\rangle .
\end{aligned}
$$

The matrix elements can be obtained directly from the correlation double one-forms and two-forms, by

$$
\begin{aligned}
& C_{V, m n}(\omega)=\iint_{V_{1} V_{2}} \mathcal{V}_{m}{ }^{*} \wedge \boldsymbol{\Gamma}_{E}\left(\boldsymbol{r}_{1}, \boldsymbol{r}_{2}, \omega\right) \wedge \mathcal{V}_{n}, \\
& C_{I, m n}(\omega)=\iint_{V_{1}^{\prime} V_{2}^{\prime}} \mathcal{U}_{m}^{\prime}{ }^{*} \wedge \boldsymbol{\Gamma}_{J}\left(\boldsymbol{r}_{1}^{\prime}, \boldsymbol{r}_{2}^{\prime}, \omega\right) \wedge \mathcal{U}_{n}^{\prime} .
\end{aligned}
$$

The correlation matrix of generalized voltages $\mathbf{C}_{V}(\omega)$, given by its coefficients $C_{V, m n}(\omega)$ can be related to the matrix of generalized currents $\mathbf{C}_{I}(\omega)$, given by $C_{I, m n}(\omega)$ using Eq. (40). Hence, we obtain

$\mathbf{C}_{V}(\omega)=\mathbf{Z}(\omega) \mathbf{C}_{I}(\omega) \mathbf{Z}^{\dagger}(\omega)$,

By this we have established a direct connection between the correlation matrix of the sources and the correlations of the observed field.

\section{Analytic calculations}

In order to show, that exterior calculus could improve our understanding of electromagnetic fields in general, and stochastic electromagnetic fields in particular, we perform analytic calculations on the stochastic emission of two Hertzian dipoles.

The Green's double one-form $\mathcal{G}$, we introduced in Eq. (14), can be expanded as

$$
\begin{aligned}
\mathcal{G} & =G_{x x^{\prime}} \mathrm{d} x \otimes \mathrm{d} x^{\prime}+G_{x y^{\prime}} \mathrm{d} x \otimes \mathrm{d} y^{\prime}+G_{x z^{\prime}} \mathrm{d} x \otimes \mathrm{d} z^{\prime} \\
& +G_{y x^{\prime}} \mathrm{d} y \otimes \mathrm{d} x^{\prime}+G_{y y^{\prime}} \mathrm{d} y \otimes \mathrm{d} y^{\prime}+G_{y z^{\prime}} \mathrm{d} y \otimes \mathrm{d} z^{\prime} \\
& +G_{z x^{\prime}} \mathrm{d} z \otimes \mathrm{d} x^{\prime}+G_{z y^{\prime}} \mathrm{d} z \otimes \mathrm{d} y^{\prime}+G_{z z^{\prime}} \mathrm{d} z \otimes \mathrm{d} z^{\prime},
\end{aligned}
$$

when choosing a Cartesian coordinate system. For the sake of a more streamlined notation, we write

$$
\left|\boldsymbol{r}-\boldsymbol{r}^{\prime}\right|=\sqrt{\left(x-x^{\prime}\right)^{2}+\left(y-y^{\prime}\right)^{2}+\left(z-z^{\prime}\right)^{2}},
$$

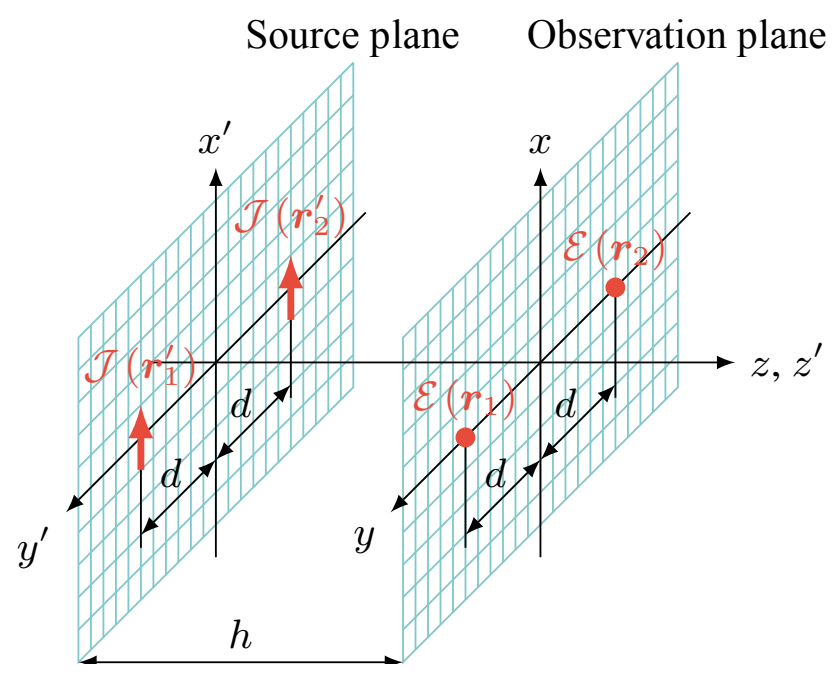

Figure 1. Setup for analytic calculations.

instead of writing down each spatial component separately. Let us also introduce the functions $g_{1}\left(\boldsymbol{r}, \boldsymbol{r}^{\prime}\right)$ and $g_{2}\left(\boldsymbol{r}, \boldsymbol{r}^{\prime}\right)$ as part of the Green's double one-form as

$g_{1}\left(\boldsymbol{r}, \boldsymbol{r}^{\prime}\right)=-\frac{3 j}{\left|\boldsymbol{r}-\boldsymbol{r}^{\prime}\right|^{5}}-\frac{3 k}{\left|\boldsymbol{r}-\boldsymbol{r}^{\prime}\right|^{4}}+\frac{j k^{2}}{\left|\boldsymbol{r}-\boldsymbol{r}^{\prime}\right|^{3}}$,

$g_{2}\left(\boldsymbol{r}, \boldsymbol{r}^{\prime}\right)=-\frac{j k^{2}}{\left|\boldsymbol{r}-\boldsymbol{r}^{\prime}\right|}+\frac{k}{\left|\boldsymbol{r}-\boldsymbol{r}^{\prime}\right|^{2}}+\frac{j}{\left|\boldsymbol{r}-\boldsymbol{r}^{\prime}\right|^{3}}$.

With Eq. (47), (48) and (49), the coefficients of the Green's double one-form from Eq. (46) can be expressed as

$$
\begin{aligned}
& G_{x x^{\prime}}=\frac{Z_{0} e^{-j k\left|\boldsymbol{r}-\boldsymbol{r}^{\prime}\right|}}{4 \pi k}\left[g_{1} \cdot\left(x-x^{\prime}\right)^{2}+g_{2}\right], \\
& G_{x y^{\prime}}=G_{y x^{\prime}}=\frac{Z_{0} e^{-j k\left|\boldsymbol{r}-\boldsymbol{r}^{\prime}\right|}}{4 \pi k} g_{1} \cdot\left(x-x^{\prime}\right)\left(y-y^{\prime}\right), \\
& G_{x z^{\prime}}=G_{z x^{\prime}}=\frac{Z_{0} e^{-j k\left|\boldsymbol{r}-\boldsymbol{r}^{\prime}\right|}}{4 \pi k} g_{1} \cdot\left(x-x^{\prime}\right)\left(z-z^{\prime}\right), \\
& G_{y y^{\prime}}=\frac{Z_{0} e^{-j k\left|\boldsymbol{r}-\boldsymbol{r}^{\prime}\right|}}{4 \pi k}\left[g_{1} \cdot\left(y-y^{\prime}\right)^{2}+g_{2}\right], \\
& G_{y z^{\prime}}=G_{z y^{\prime}}=\frac{Z_{0} e^{-j k\left|\boldsymbol{r}-\boldsymbol{r}^{\prime}\right|}}{4 \pi k} g_{1} \cdot\left(y-y^{\prime}\right)\left(z-z^{\prime}\right), \\
& G_{z z^{\prime}}=\frac{Z_{0} e^{-j k\left|\boldsymbol{r}-\boldsymbol{r}^{\prime}\right|}}{4 \pi k}\left[g_{1} \cdot\left(z-z^{\prime}\right)^{2}+g_{2}\right] .
\end{aligned}
$$

We consider two Hertzian dipoles oriented in $x$ direction, excited with random currents, $I_{1}(\omega)$ and $I_{2}(\omega)$. The model of two Hertzian dipoles is simple enough, to allow for an analytic treatment.

A sketch of the setup we use for our analytic considerations is given by Fig. 1 .

The distance between the dipoles is $2 d$ in $y$ direction. Thus, we define the source-current density as

$\mathcal{J}=I_{1}(\omega) l \delta(x) \delta(y-d) \delta(z) \mathrm{d} y \wedge \mathrm{d} z$ 


$$
+I_{2}(\omega) l \delta(x) \delta(y+d) \delta(z) \mathrm{d} y \wedge \mathrm{d} z .
$$

According to Eq. (14), we can obtain the electric field, excited from this current density by

$$
\begin{aligned}
\mathcal{E} & =\int_{V^{\prime}} \mathcal{G} \wedge \mathcal{J}^{\prime}=\int_{V^{\prime}}\left(G_{x x^{\prime}} \mathrm{d} x+G_{y x^{\prime}} \mathrm{d} y+G_{z x^{\prime}} \mathrm{d} z\right) \times \\
& \times I_{1}(\omega) l \delta\left(x^{\prime}\right) \delta\left(y^{\prime}-d\right) \delta\left(z^{\prime}\right) \mathrm{d} x^{\prime} \wedge \mathrm{d} y^{\prime} \wedge \mathrm{d} z^{\prime} \\
& +\int_{V^{\prime}}\left(G_{x x^{\prime}} \mathrm{d} x+G_{y x^{\prime}} \mathrm{d} y+G_{z x^{\prime}} \mathrm{d} z\right) \times \\
& \times I_{2}(\omega) l \delta\left(x^{\prime}\right) \delta\left(y^{\prime}+d\right) \delta\left(z^{\prime}\right) \mathrm{d} x^{\prime} \wedge \mathrm{d} y^{\prime} \wedge \mathrm{d} z^{\prime} .
\end{aligned}
$$

The Dirac delta functions within $\mathcal{J}$ act as a spatial shift, such that we need to evaluate the coefficients for the Green's double one-form only for specific source points,

$\boldsymbol{r}_{1}^{\prime}=[0, d, 0]^{T}$,

$\boldsymbol{r}_{2}^{\prime}=[0,-d, 0]^{T}$.

We introduce the following functions

$$
\begin{aligned}
g_{1( \pm)} & =-\frac{3 j}{\left(\sqrt{x^{2}+(y \pm d)^{2}+z^{2}}\right)^{5}} \\
& -\frac{3 k}{\left(\sqrt{x^{2}+(y \pm d)^{2}+z^{2}}\right)^{4}} \\
& +\frac{j k^{2}}{\left(\sqrt{x^{2}+(y \pm d)^{2}+z^{2}}\right)^{3}}, \\
g_{2( \pm)} & =\frac{-j k^{2}}{\sqrt{x^{2}+(y \pm d)^{2}+z^{2}}}+\frac{k}{\left(\sqrt{x^{2}+(y \pm d)^{2}+z^{2}}\right)^{2}} \\
& +\frac{j}{\left(\sqrt{x^{2}+(y \pm d)^{2}+z^{2}}\right)^{3}},
\end{aligned}
$$

where $g_{i_{(-)}}$is given by $g_{i}\left(\boldsymbol{r}, \boldsymbol{r}_{1}^{\prime}\right)$, and $g_{i(+)}$ is given by $g_{i}\left(\boldsymbol{r}, \boldsymbol{r}_{2}^{\prime}\right)$.

Using the shorthand functions $g_{i(-)}$ and $g_{i(+)}$, we obtain the solution for the electric field one-form in the whole observation domain as

$$
\begin{aligned}
\mathcal{E} & =\frac{I_{1}(\omega) l Z_{0} e^{-j k \sqrt{x^{2}+(y-d)^{2}+z^{2}}}}{4 \pi k} \times \\
& \times\left[g_{1(-)}\left(x^{2} \mathrm{~d} x+x(y-d) \mathrm{d} y+x z \mathrm{~d} z\right)+g_{2(-)} \mathrm{d} x\right] \\
& +\frac{I_{2}(\omega) l Z_{0} e^{-j k \sqrt{x^{2}+(y+d)^{2}+z^{2}}}}{4 \pi k} \times \\
& \times\left[g_{1_{(+)}}\left(x^{2} \mathrm{~d} x+x(y+d) \mathrm{d} y+x z \mathrm{~d} z\right)+g_{2_{(+)}} \mathrm{d} x\right] .
\end{aligned}
$$

The current density two-forms at locations $\boldsymbol{r}_{1}^{\prime}$ and $\boldsymbol{r}_{2}^{\prime}$ are simply given by

$$
\begin{aligned}
& \mathcal{J}\left(\boldsymbol{r}_{1}^{\prime}\right)=I_{1}(\omega) \mathrm{d} y \wedge \mathrm{d} z, \\
& \mathcal{J}\left(\boldsymbol{r}_{2}^{\prime}\right)=I_{2}(\omega) \mathrm{d} y \wedge \mathrm{d} z .
\end{aligned}
$$

With this, we are able to calculate the correlation matrix elements for the source currents,

$$
\begin{aligned}
\Gamma_{J}\left(\boldsymbol{r}_{1}^{\prime}, \boldsymbol{r}_{1}^{\prime}, \omega\right) & =\left\langle\mathcal{J}\left(\boldsymbol{r}_{1}^{\prime}\right) \otimes \mathcal{J}^{*}\left(\boldsymbol{r}_{1}^{\prime}\right)\right\rangle \\
& =\left\langle I_{1}(\omega) I_{1}^{*}(\omega)\right\rangle \mathrm{d} y_{1}^{\prime} \wedge \mathrm{d} z_{1}^{\prime} \otimes \mathrm{d} y_{1}^{\prime} \wedge \mathrm{d} z_{1}^{\prime}, \\
\Gamma_{J}\left(\boldsymbol{r}_{1}^{\prime}, \boldsymbol{r}_{2}^{\prime}, \omega\right) & =\left\langle\mathcal{J}\left(\boldsymbol{r}_{1}^{\prime}\right) \otimes \mathcal{J}^{*}\left(\boldsymbol{r}_{2}^{\prime}\right)\right\rangle \\
& =\left\langle I_{1}(\omega) I_{2}^{*}(\omega)\right\rangle \mathrm{d} y_{1}^{\prime} \wedge \mathrm{d} z_{1}^{\prime} \otimes \mathrm{d} y_{2}^{\prime} \wedge \mathrm{d} z_{2}^{\prime}, \\
\Gamma_{J}\left(\boldsymbol{r}_{2}^{\prime}, \boldsymbol{r}_{1}^{\prime}, \omega\right) & =\left\langle\mathcal{J}\left(\boldsymbol{r}_{2}^{\prime}\right) \otimes \mathcal{J}^{*}\left(\boldsymbol{r}_{1}^{\prime}\right)\right\rangle \\
& =\left\langle I_{2}(\omega) I_{1}^{*}(\omega)\right\rangle \mathrm{d} y_{2}^{\prime} \wedge \mathrm{d} z_{2}^{\prime} \otimes \mathrm{d} y_{1}^{\prime} \wedge \mathrm{d} z_{1}^{\prime}, \\
\Gamma_{J}\left(\boldsymbol{r}_{2}^{\prime}, \boldsymbol{r}_{2}^{\prime}, \omega\right) & =\left\langle\mathcal{J}\left(\boldsymbol{r}_{2}^{\prime}\right) \otimes \mathcal{J}^{*}\left(\boldsymbol{r}_{2}^{\prime}\right)\right\rangle \\
& =\left\langle I_{2}(\omega) I_{2}^{*}(\omega)\right\rangle \mathrm{d} y_{2}^{\prime} \wedge \mathrm{d} z_{2}^{\prime} \otimes \mathrm{d} y_{2}^{\prime} \wedge \mathrm{d} z_{2}^{\prime} .
\end{aligned}
$$

The currents $I_{1}(\omega)$ and $I_{2}(\omega)$ are chosen to be uncorrelated, i.e. $\left\langle I_{1}(\omega) I_{1}^{*}(\omega)\right\rangle=\left\langle I_{2}(\omega) I_{2}^{*}(\omega)\right\rangle=1$ and $\left\langle I_{1}(\omega) I_{2}^{*}(\omega)\right\rangle=$ $\left\langle I_{2}(\omega) I_{1}^{*}(\omega)\right\rangle=0$. The correlations double one-forms of the excited fields are continuous in space, just like the correlation two-forms of the source currents. But in contrast to the sources, defined by Dirac delta functions, the fields do not vanish everywhere, except for the observation points. Hence, we want to observe the correlations of the electric field only at two distinct locations at some distance $h$ from the Hertzian dipoles in $z$ direction, in order to obtain finite dimensional correlation matrices, which we can compare to the source correlations.

The locations of our observation points are given by the coordinates,

$\boldsymbol{r}_{1}=[0, d, h]^{T}$,
$\boldsymbol{r}_{2}=[0,-d, h]^{T}$.

Figure 1 also shows how source and observation plane are aligned.

The electric fields at locations $\boldsymbol{r}_{1}$ and $\boldsymbol{r}_{2}$ are given by

$$
\begin{aligned}
\mathcal{E}\left(\boldsymbol{r}_{1}\right) & =\frac{I_{1}(\omega) l Z_{0} e^{-j k h}}{4 \pi k} g_{2(-)}\left(\boldsymbol{r}_{1}\right) \mathrm{d} x \\
& +\frac{I_{2}(\omega) l Z_{0} e^{-j k \sqrt{4 d^{2}+h^{2}}}}{4 \pi k} g_{2(+)}\left(\boldsymbol{r}_{1}\right) \mathrm{d} x,
\end{aligned}
$$

and

$$
\begin{aligned}
\mathcal{E}\left(\boldsymbol{r}_{2}\right) & =\frac{I_{1}(\omega) l Z_{0} e^{-j k \sqrt{4 d^{2}+h^{2}}}}{4 \pi k} g_{2(-)}\left(\boldsymbol{r}_{2}\right) \mathrm{d} x \\
& +\frac{I_{2}(\omega) l Z_{0} e^{-j k h}}{4 \pi k} g_{2(+)}\left(\boldsymbol{r}_{2}\right) \mathrm{d} x .
\end{aligned}
$$

From this, we can evaluate the matrix elements of the correlations of the electric field at locations $\boldsymbol{r}_{1}$ and $\boldsymbol{r}_{2}$. The auto- 
and cross-correlation spectra are given by

$$
\begin{aligned}
\boldsymbol{\Gamma}_{E}\left(\boldsymbol{r}_{1}, \boldsymbol{r}_{1}, \omega\right) & =\left\langle\mathcal{E}\left(\boldsymbol{r}_{1}\right) \otimes \mathcal{E}^{*}\left(\boldsymbol{r}_{1}\right)\right\rangle \\
& =\frac{l^{2} Z_{0}^{2}}{16 \pi^{2} k^{2}}\left|g_{2(-)}\left(\boldsymbol{r}_{1}\right)\right|^{2} \mathrm{~d} x_{1} \otimes \mathrm{d} x_{1} \\
& +\frac{l^{2} Z_{0}^{2}}{16 \pi^{2} k^{2}}\left|g_{2(+)}\left(\boldsymbol{r}_{1}\right)\right|^{2} \mathrm{~d} x_{1} \otimes \mathrm{d} x_{1},
\end{aligned}
$$

$$
\begin{aligned}
\boldsymbol{\Gamma}_{E}\left(\boldsymbol{r}_{1}, \boldsymbol{r}_{2}, \omega\right) & =\left\langle\mathcal{E}\left(\boldsymbol{r}_{1}\right) \otimes \mathcal{E}^{*}\left(\boldsymbol{r}_{2}\right)\right\rangle \\
& =\frac{l^{2} Z_{0}^{2} e^{-j k\left(h-\sqrt{4 d^{2}+h^{2}}\right)}}{16 \pi^{2} k^{2}} \times \\
& \times g_{2(-)}\left(\boldsymbol{r}_{1}\right) g_{2(-)}^{*}\left(\boldsymbol{r}_{2}\right) \mathrm{d} x_{1} \otimes \mathrm{d} x_{2} \\
& +\frac{l^{2} Z_{0}^{2} e^{-j k\left(\sqrt{4 d^{2}+h^{2}}-h\right)}}{16 \pi^{2} k^{2}} \times \\
& \times g_{2(+)}\left(\boldsymbol{r}_{1}\right) g_{2(+)}^{*}\left(\boldsymbol{r}_{2}\right) \mathrm{d} x_{1} \otimes \mathrm{d} x_{2},
\end{aligned}
$$

$$
\begin{aligned}
\boldsymbol{\Gamma}_{E}\left(\boldsymbol{r}_{2}, \boldsymbol{r}_{1}, \omega\right) & =\left\langle\mathcal{E}\left(\boldsymbol{r}_{2}\right) \otimes \mathcal{E}^{*}\left(\boldsymbol{r}_{1}\right)\right\rangle \\
& =\frac{l^{2} Z_{0}^{2} e^{-j k\left(\sqrt{4 d^{2}+h^{2}}-h\right)}}{16 \pi^{2} k^{2}} \times \\
& \times g_{2(-)}\left(\boldsymbol{r}_{2}\right) g_{2(-)}^{*}\left(\boldsymbol{r}_{1}\right) \mathrm{d} x_{2} \otimes \mathrm{d} x_{1} \\
& +\frac{l^{2} Z_{0}^{2} e^{-j k\left(h-\sqrt{4 d^{2}+h^{2}}\right)}}{16 \pi^{2} k^{2}} \times \\
& \times g_{2(+)}\left(\boldsymbol{r}_{2}\right) g_{2(+)}^{*}\left(\boldsymbol{r}_{1}\right) \mathrm{d} x_{2} \otimes \mathrm{d} x_{1},
\end{aligned}
$$

$$
\begin{aligned}
\boldsymbol{\Gamma}_{E}\left(\boldsymbol{r}_{2}, \boldsymbol{r}_{2}, \omega\right) & =\left\langle\mathcal{E}\left(\boldsymbol{r}_{2}\right) \otimes \mathcal{E}^{*}\left(\boldsymbol{r}_{2}\right)\right\rangle \\
& =\frac{l^{2} Z_{0}^{2}}{16 \pi^{2} k^{2}}\left|g_{2(-)}\left(\boldsymbol{r}_{2}\right)\right|^{2} \mathrm{~d} x_{2} \otimes \mathrm{d} x_{2} \\
& +\frac{l^{2} Z_{0}^{2}}{16 \pi^{2} k^{2}}\left|g_{2(+)}\left(\boldsymbol{r}_{2}\right)\right|^{2} \mathrm{~d} x_{2} \otimes \mathrm{d} x_{2} .
\end{aligned}
$$

This shows that the excited fields at a distance are no longer uncorrelated, since the radiation from the source at $\boldsymbol{r}_{1}^{\prime}$ also affects the field observed in $\boldsymbol{r}_{2}$. As expected, the magnitude of the cross-correlation spectra increases compared to the autocorrelation spectra with increasing observation distance. The analytic expression presented here describes how our ability to distinguish between the two dipole sources recedes as the observation distance $h$ increases while $d$ remains constant. The correlation matrix of the field samples exhibits block Toeplitz character in the far-field (Russer et al., 2015a).

\section{Conclusions}

We have revisited the the theoretic description of stochastic electromagnetic fields and introduced exterior calculus for their description. The method of moments was applied within the framework of differential forms in order to obtain equations which can be treated numerically. These equations are equivalent to those obtained from traditional vector calculus. The main advantage, however, of the formulation presented over the traditional formulation, given in terms of the vector calculus notation, is the formulation's independence of any choice of a particular coordinate system. Other benefits, like the graphical representation of stochastic field forms, may be worth to be further explored. We derived the correlation matrix of stochastic generalized voltages from the correlation matrix of generalized currents. The involved linear operators, i.e. the impedance matrices $\mathbf{Z}(\omega)$ are the same as calculated for deterministic problems. The impedance matrices, in turn only depend on the basis functions and the Green's dyadic for the considered field problem. Thus, the transformation matrix computed for deterministic field problems can also be applied to noisy field problems (Russer and Russer, 2015). This facilitates the use of numerical tools, developed for solving deterministic field problems, to treat, in combination with the method presented, problems related to stochastic electromagnetic fields. Finally, we have given an analytic example for calculating field-field-correlations at distinct observation points in free space, using the framework of exterior calculus on stochastic electromagnetic fields. While the example of two dipole sources presented here is simple, it can be easily extended to the case of a two-dimensional source (dipole) array, representing for example the currents on a printed circuit board (PCB). This allows the investigation of noisy electromagnetic field propagation in complex environments or the investigation of inverse problems such as stochastic source localization based on near-field measurements.

Competing interests. The authors declare that they have no conflict of interest.

Acknowledgements. This work was supported by the European Union's Horizon 2020 research and innovation programme under grant no. 664828 (NEMF21).

Edited by: R. Schuhmann

Reviewed by: two anonymous referees

\section{References}

Arnaut, L. R. and Obiekezie, C. S.: Comparison of Complex Principal and Independent Components for QuasiGaussian Radiated Emissions From Printed Circuit Boards, IEEE T. Electromagn. C., 56, 1598-1603, https://doi.org/10.1109/TEMC.2014.2343912, 2014.

Baev, A., Gorbunova, A., Konovalyuk, M., Kuznetsov, Y., and Russer, J. A.: Planar stochastic sources localization algorithm in EMC problems, in: International Conference on Electromagnetics in Advanced Applications (ICEAA), 9-13 September 2013, Torino, Italy, 440-443, 2013. 
Cartan, E.: Systèmes Différentielles Extérieurs et leurs applications géométriques, Hermann, Paris, 1945.

Felsen, L. B., Mongiardo, M., and Russer, P.: Electromagnetic Field Computation by Network Methods, Springer-Verlag, Berlin, Heidelberg, New York, 2009.

Gibson, W. C.: The Method of Moments in Electromagnetics, Chapman \& Hall/CRC, Taylor \& Francis Group, 2008.

Gorbunova, A., Baev, A., Konovalyuk, M., Kuznetsov, Y., and Russer, J. A.: Stochastic EMI sources localization algorithm based on time domain planar near-field scanning, in: 2013 International Symposium on Electromagnetic Compatibility (EMC EUROPE), 2-6 September 2013, Brugge, Belgium, 972-976, 2013.

Gradoni, G., Creagh, S. C., Tanner, G., Smartt, C., and Thomas, D. W. P.: A phase-space approach for propagating field-field correlation functions, New J. Phys., 17, 093027, https://doi.org/10.1088/1367-2630/17/9/093027, 2015.

Grassmann, H. and Kannenberg, L.: A New Branch of Mathematics: The "Ausdehnungslehre" of 1844 and Other Works, Open Court Publishing, Chicago, 1995.

Haider, M. and Russer, J. A.: Principal Component Analysis for Efficient Characterization of Stochastic Electromagnetic Fields, International Journal of Numerical Modelling: Electronic Networks, Devices and Fields, https://doi.org/10.1002/jnm.2246, in press, 2017.

Harrington, R. F.: Field Computation by Moment Methods, IEEE Press, San Francisco, 1968.

Kuznetsov, Y., Baev, A., Gorbunova, A., Konovalyuk, M., Thomas, D., Smartt, C., Baharuddin, M. H., Russer, J. A., and Russer, P.: Localization of the Equivalent Sources on the PCB Surface by Using Ultra-Wideband Time Domain Near-Field Measurements, in: 2016 International Symposium on Electromagnetic Compatibility (EMC EUROPE), 5-9 September 2016, Wroclaw, Poland, https://doi.org/10.1109/EMCEurope.2016.7739184, 2016.

Lang, S.: Fundamentals of Differential Geometry, in: Graduate Texts in Mathematics, Vol. 191, Springer-Verlag New York Berlin Heidelberg, 1999.

Lindell, I. V.: Differential Forms in Electromagnetics, IEEE Press, New York, 2004.

Nossek, J. A., Russer, P., Noll, T., Mezghani, A., Ivrlač, M. T., Korb, M., Mukhtar, F., Yordanov, H., and Russer, J. A.: Chip-to-Chip and On-Chip Communications, in: Ultra-Wideband Radio Technologies for Communications, Localization and Sensor Applications, edited by: Thomä, R., Knöchel, R. H., Sachs, J., Willms, I., and Zwick, T., InTech, 75-108, https://doi.org/10.5772/52981, 2013.

Russer, J. A. and Russer, P.: An Efficient Method for Computer Aided Analysis of Noisy Electromagnetic Fields, in: 2011 IEEE MTT-S International Microwave Symposium Digest (MTT), 5-10 June 2011, Baltimore, MD, USA, 1-4, https://doi.org/10.1109/MWSYM.2011.5972651, 2011a.

Russer, J. A. and Russer, P.: Imaging of Sources of Radiated Electromagnetic Interference, Frequenz, 65, 261-265, https://doi.org/10.1515/FREQ.2011.038, 2011b.

Russer, J. A. and Russer, P.: Network Methods Applied to the Computation of Stochastic Electromagnetic Fields, in: 2011 International Conference on Electromagnetics in Advanced Applications (ICEAA), 12-16 September 2011, Torino, Italy, 11521155, https://doi.org/10.1109/ICEAA.2011.6046513, $2011 \mathrm{c}$.
Russer, J. A. and Russer, P.: Stochastic Electromagnetic Fields, in: 2011 German Microwave Conference (GeMIC), 14-16 March 2011, Darmstadt, Germany, 2011d.

Russer, J. A. and Russer, P.: Modeling of Noisy EM Field Propagation Using Correlation Information, IEEE T. Microw. Theory, 63, 76-89, https://doi.org/10.1109/TMTT.2014.2376962, 2015.

Russer, J. A., Mukhtar, F., Filonik, O., Scarpa, G., and Russer, P.: Modelling of Noisy EM Field Propagation Using Correlation Information of Sampled Data, in: 2014 International Conference on Numerical Electromagnetic Modeling and Optimization for RF, Microwave, and Terahertz Applications (NEMO), 1-4, https://doi.org/10.1109/NEMO.2014.6995713, 2014.

Russer, J. A., Gradoni, G., Tanner, G., Creagh, S. C., Thomas, D., Smartt, C., and Russer, P.: Evolution of transverse correlation in stochastic electromagnetic fields, in: 2015 IEEE MTT-S International Microwave Symposium (IMS), 17-22 May 2015, Phoenix, Arizona, https://doi.org/10.1109/MWSYM.2015.7166953, 2015a.

Russer, J. A., Russer, P., Konovalyuk, M., Gorbunova, A., Baev, A., and Kuznetsov, Y.: Analysis of Cyclostationary Stochastic Electromagnetic Fields, in: International Conference on Electromagnetics in Advanced Applications (ICEAA), 7-11 September 2015, Torino, Italy, 1452-1455, https://doi.org/10.1109/ICEAA.2015.7297359, 2015b.

Russer, J. A., Russer, P., Konovalyuk, M., Gorbunova, A., Baev, A., and Kuznetsov, Y.: Near-Field Propagation of Cyclostationary Stochastic Electromagnetic Fields, in: International Conference on Electromagnetics in Advanced Applications (ICEAA), 7-11 September 2015, Torino, Italy, https://doi.org/10.1109/ICEAA.2015.7297360, 1456-1459, 2015c.

Russer, J. A., Uddin, N., Awny, A. S., Thiede, A., and Russer, P.: Near-field measurement of stochastic electromagnetic fields, Electromagnetic Compatibility Magazine, IEEE, 4, 79-85, https://doi.org/10.1109/MEMC.2015.7336761, 2015d.

Russer, J. A., Cangellaris, A., and Russer, P.: Correlation Transmission Line Matrix (CTLM) modeling of stochastic electromagnetic fields, in: 2016 IEEE MTT$\mathrm{S}$ International Microwave Symposium (IMS), 1-4, https://doi.org/10.1109/MWSYM.2016.7540091, 2016.

Russer, P.: Electromagnetics, Microwave Circuit and Antenna Design for Communications Engineering, 2nd Edn., Artech House, Boston, 2006.

Warnick, K. F. and Arnold, D. V.: Electromagnetic Green functions using differential forms, J. Electromagnet. Waves, 10, 427-438, 1996.

Warnick, K. F. and Russer, P.: Two, Three and Four-Dimensional Electromagnetics Using Differential Forms, Turk. J. Electr. Eng. Co., 14, 153-172, 2006.

Warnick, K. F. and Russer, P.: Differential Forms and Electromagnetic Field Theory (Invited Paper), Progress In Electromagnetics Research, 148, 83-112, https://doi.org/10.2528/PIER14063009, 2014. 